\title{
Research Article \\ Synchronization for an Array of Coupled Cohen-Grossberg Neural Networks with Time-Varying Delay
}

\author{
Haitao Zhang, ${ }^{1}$ Tao $\mathrm{Li}^{2}{ }^{2}$ and Shumin Fei ${ }^{1}$ \\ ${ }^{1}$ Key Laboratory of Measurement and Control of CSE, School of Automation, Southeast University, \\ Ministry of Education, Nanjing 210096, China \\ 2 School of Automation Engineering, Nanjing University of Aeronautics and Astronautics, \\ Nanjing 2010016, China
}

Correspondence should be addressed to Haitao Zhang, zhtnanjing@126.com

Received 23 November 2010; Accepted 9 March 2011

Academic Editor: Bin Liu

Copyright (C) 2011 Haitao Zhang et al. This is an open access article distributed under the Creative Commons Attribution License, which permits unrestricted use, distribution, and reproduction in any medium, provided the original work is properly cited.

This paper makes some great attempts to investigate the global exponential synchronization for arrays of coupled delayed Cohen-Grossberg neural networks with both delayed coupling and one single delayed one. By resorting to free-weighting matrix and Kronecker product techniques, two novel synchronization criteria via linear matrix inequalities (LMIs) are presented based on convex combination, in which these conditions are heavily dependent on the bounds of both the delay and its derivative. Owing to that the addressed system can include some famous neural network models as the special cases, the proposed methods can extend and improve those earlier reported ones. The efficiency and applicability of the presented conditions can be demonstrated by two numerical examples with simulations.

\section{Introduction}

In recent years, synchronization of various chaotic systems has gained considerable attention since the pioneering works of Pecora and Carroll [1,2]. It is widely known that many benefits of having synchronization or chaos synchronization can be existent in various engineering fields such as secure communication, image processing, and harmonic oscillation generation. Thus recently, the problem on synchronization in chaotic systems has been extensively studied owing to its potential applications in many engineering areas. Especially, since chaos synchronization in arrays of linearly coupled dynamical systems was firstly studied in [3], arrays of coupled systems including delayed chaotic ones have received much attention as 
they can exhibit some interesting phenomena $[4,5]$, and a great number of elegant results have been derived [6-28].

As a typical complex systems, delayed neural networks (DNNs) have been verified to exhibit some complex and unpredictable behaviors such as stable equilibria, periodic oscillations, bifurcation, and chaotic attractors. Thus recently, chaos synchronization for arrays of coupled DNNs have been discussed and some elegant results have been propose [12-28]. The global synchronization of linearly coupled DNNs with delayed coupling was investigated in [12], in which the dynamical behavior of the uncoupled system could be chaotic or others. The authors in [13] have considered the robust synchronization of coupled DNNs under general impulsive control. In [14], this paper has proposed an adaptive procedure to the synchronization for coupled identical Yang-Yang fuzzy DNNs based on one simple adaptive controller. In [15], with all the parameters unknown, the authors focused on the robust synchronization between two coupled DNNs that were linearly and unidirectionally coupled, in which neither symmetry nor negative (positive) definiteness of the coupling matrix were required. However, those above-mentioned results were presented in terms of some complicated inequalities, which makes them uneasily checked and applied to real ceases by the most recently developed algorithms. By employing Kronecker product and LMI technique, the global synchronization and cluster one have been studied for DNNs with couplings, and some easy-to-test sufficient conditions have been obtained [16-25, 28]. Yet, the system forms addressed in [16-25] seemed simple and some improved techniques have not been utilized to reduce the conservatism, which make the above-mentioned results inapplicable to tackle DNNs of more general forms.

The Cohen-Grossberg neural network (CGNN) model, first proposed by Cohen and Grossberg in 1983 [29], has recently gained particular research attention, since it is quite general to include many famous network models as its special case and has promising application potentials for tasks of associative memory, parallel computation, and nonlinear optimization problems. Meanwhile, owing to complexity of CGNNs themselves, there were few works studying the global synchronization for the coupled delayed CGNNs, except for that some researchers have studied the slave-master synchronization for continuous CGNNs in [26, 27] and synchronization for coupled discrete delayed CGNNs in [28]. Thus, it is urgent and challenging to establish some easy-to-check and less conservative results ensuring the global synchronization of coupled continuous-time delayed CGNNs, which constitutes the main focus of this presented work.

In this paper, the global exponential synchronization of $N$ identical delayed CGNNs with both delayed coupling and one single delayed one is considered and two novel LMIbased conditions are derived by using Kronecker product technique, which has not been studied in the present literature. It shows that the chaos synchronization can be ensured by a suitable design of inner coupled linking matrix and the inner delayed linking ones. Moreover, some effective mathematical techniques are employed to reduce the conservatism. Finally, the efficiency of the derived criteria can be illustrated by utilizing two numerical examples.

Notations. $\mathbf{R}^{n}$ denotes the $n$-dimensional Euclidean space, and $\mathbf{R}^{n \times m}$ is the set of all $n \times m$ real matrices. For the symmetric matrices $X, Y, X>Y$ (respectively, $X \geq Y$ ) means that $X-Y>0(X-Y \geq 0)$ is a positive-definite (respectively, positive-semidefinite) matrix; $A^{T}$ represents the transpose of the matrix $A ; \lambda_{\max }(A), \lambda_{\min }(A)$ denote the maximum eigenvalue and minimum one of matrix $A$, respectively; $I$ represents the identity matrix of an appropriate dimension; $\left[\begin{array}{cc}X & Y \\ Y^{T} & Z\end{array}\right]=\left[\begin{array}{ll}X & Y \\ * & Z\end{array}\right]$. 


\section{Problem Formulations and Preliminaries}

Suppose the nodes are coupled with states $x_{i}(t), i \in\{1, \ldots, N\}$, then the delayed CohenGrossberg neural network models can be formulated as follows:

$$
\begin{aligned}
\dot{x}_{i}(t)= & -\alpha\left(x_{i}(t)\right)\left[\beta\left(x_{i}(t)\right)-A f\left(x_{i}(t)\right)-B f\left(x_{i}(t-\tau(t))\right)-\mathbf{I}(t)\right] \\
& +\sum_{j=1, j \neq i}^{N} l_{i j} F\left[x_{j}(t)-x_{i}(t)\right] \\
& +\sum_{j=1, j \neq i}^{N} l_{i j} K\left[x_{j}(t-\tau(t))-x_{i}(t)\right] \\
& +\sum_{j=1, j \neq i}^{N} l_{i j} J\left[x_{j}(t-\tau(t))-x_{i}(t-\tau(t))\right],
\end{aligned}
$$

in which $x_{i}(t)=\left[x_{i 1}(t), \ldots, x_{i n}(t)\right]^{T} \in \mathbf{R}^{n}$ is the state vector of the $i$ th network at time $t, \alpha\left(x_{i}\right)=$ $\operatorname{diag}\left\{\alpha_{1}\left(x_{i 1}\right), \ldots, \alpha_{n}\left(x_{\text {in }}\right)\right\}$ represents the amplification function, $\beta\left(x_{i}\right)=\left[\beta_{1}\left(x_{i 1}\right), \ldots, \beta_{n}\left(x_{\text {in }}\right)\right]^{T}$ is the behaved function, $A=\left[a_{i j}\right]_{n \times n}, B=\left[b_{i j}\right]_{n \times n}, f\left(x_{i}\right)=\left[f_{1}\left(x_{i 1}\right), \ldots, f_{n}\left(x_{i n}\right)\right]^{T} ; \mathrm{I}(t)=$ $\left[\mathbf{I}_{1}(\mathrm{t}), \ldots, \mathbf{I}_{n}(\mathrm{t})\right]^{T} \in \mathbf{R}^{n}$ is the external input vector; $F=\left[f_{i j}\right]_{n \times n}, K=\left[k_{i j}\right]_{n \times n}$, and $J=\left[j_{i j}\right]_{n \times n}$ are respectively the inner coupling matrices between the connected nodes $i$ and $j$ at times $t$ and $t-\tau(t)$.

For system (2.1), the following assumptions are introduced throughout this paper.

(A1) $\tau(t)$ denotes an interval time-varying delay satisfying

$$
0 \leq \tau_{0} \leq \tau(t) \leq \tau_{m}, \quad \dot{\tau}(t) \leq \mu<+\infty,
$$

and we set $\bar{\tau}_{m}=\tau_{m}-\tau_{0}$.

(A2) $L=\left[l_{i j}\right]_{N \times N}$ is the configuration matrix that is irreducible and satisfies

$$
l_{i j}=l_{j i}, \quad i \neq j, \quad l_{i i}=-\sum_{j=1, j \neq i}^{N} l_{i j} .
$$

Here $l_{i j}>0$ if there is a connection between node $i$ and the one $j$ and otherwise, $l_{i j}=0$.

(A3) For $i \in\{1,2, \ldots, n\}$, each $\alpha_{i}(\cdot)$ is Lipschitz continuous and there exists the positive scalars $\underline{a}_{i}, \bar{a}_{i}$ satisfying $0<\underline{a}_{i} \leq \alpha_{i}(\cdot) \leq \bar{a}_{i}$; and there exist the positive scalars $\pi_{i}, \gamma_{i}$ such that each function $\beta_{i}(\cdot)$ satisfies $0<\gamma_{i} \leq\left(\beta_{i}(x)-\beta_{i}(y) / x-y\right) \leq \pi_{i}$, and

$$
\left[\dot{\beta}_{i}(x)-\dot{\beta}_{i}(y)-\rho_{i}^{-}(x-y)\right]\left[\dot{\beta}_{i}(x)-\dot{\beta}_{i}(y)-\rho_{i}^{+}(x-y)\right] \leq 0 \quad \forall x, y \in \mathbf{R}, i=1, \ldots, n,
$$


in which $\rho_{i}^{-}, \rho_{i}^{+}$are given constants. Here we set $\Lambda=\operatorname{diag}\left\{\underline{a}_{1}, \ldots, \underline{a}_{n}\right\}, \Psi=\operatorname{diag}\left\{\bar{a}_{1}, \ldots, \bar{a}_{n}\right\}$, $\Gamma=\operatorname{diag}\left\{\gamma_{1}, \ldots, \gamma_{n}\right\}, \Pi=\operatorname{diag}\left\{\pi_{1}, \ldots, \pi_{n}\right\}$, and

$$
\begin{array}{ll}
\Lambda_{1}=\operatorname{diag}\left\{\gamma_{1} \pi_{1}, \ldots, \gamma_{n} \pi_{n}\right\}, & \Lambda_{2}=\operatorname{diag}\left\{\frac{\pi_{1}+\gamma_{1}}{2}, \ldots, \frac{\pi_{n}+\gamma_{n}}{2}\right\} \\
\Upsilon_{1}=\operatorname{diag}\left\{\rho_{1}^{+} \rho_{1}^{-}, \ldots, \rho_{n}^{+} \rho_{n}^{-}\right\}, & \Upsilon_{2}=\operatorname{diag}\left\{\frac{\rho_{1}^{+}+\rho_{1}^{-}}{2}, \ldots, \frac{\rho_{n}^{+}+\rho_{n}^{-}}{2}\right\} .
\end{array}
$$

(A4) For any $\alpha, \beta \in \mathbf{R}$, and $\rho_{i}^{-}, \rho_{i}^{+}$for $i \in\{1,2, \ldots, n\}$, the activation function $f_{i}(\cdot)$ satisfies

$$
\left[f_{i}(\alpha)-f_{i}(\beta)-\sigma_{i}^{+}(\alpha-\beta)\right]\left[f_{i}(\alpha)-f_{i}(\beta)-\sigma_{i}^{-}(\alpha-\beta)\right] \leq 0 \text {. }
$$

Here we denote $\Sigma_{1}=\operatorname{diag}\left\{\sigma_{1}^{+} \sigma_{1}^{-}, \ldots, \sigma_{n}^{+} \sigma_{n}^{-}\right\}$and $\Sigma_{2}=\operatorname{diag}\left\{\left(\sigma_{1}^{+}+\sigma_{1}^{-}\right) / 2, \ldots,\left(\sigma_{n}^{+}+\sigma_{n}^{-}\right) / 2\right\}$.

Remark 2.1. In (A3), the assumption on the derivative of $\beta\left(x_{i}\right)$ in (2.4) is reasonable and does not result in the conservatism in many cases such as that, choosing the appropriate scalars $a, b, c$, the function $\beta\left(x_{i}\right)$ can be expressed as $a x_{i}, a x_{i}+b \sin \left(x_{i}\right), a x_{i}+b \sin ^{2}\left(x_{i}\right), a x_{i}+c \cos \left(x_{i}\right)$, $a x_{i}+c \cos ^{3}\left(x_{i}\right), a x_{i}+c \tanh \left(x_{i}\right)$, respectively. Moreover, the activation functions in system (2.1) can be of general description and those present ones in [22-26, 29] are just special cases of the system (2.1).

Based on assumption(A2), system (2.1) can be rewritten as the following forms:

$$
\begin{aligned}
\dot{x}_{i}(t)= & -\alpha\left(x_{i}(t)\right)\left[\beta\left(x_{i}(t)\right)-A f\left(x_{i}(t)\right)-B f\left(x_{i}(t-\tau(t))\right)-\mathrm{I}(t)\right]-l_{i i} K\left[x_{i}(t-\tau(t))-x_{i}(t)\right] \\
& +\sum_{j=1}^{N} l_{i j} F x_{j}(t)+\sum_{j=1}^{N} l_{i j}(K+J) x_{j}(t-\tau(t)) .
\end{aligned}
$$

To address the problem, we denote the set $\mathcal{S}=\left\{\mathrm{x}(s)=\left[x_{1}^{T}(s), \ldots, x_{N}^{T}(s)\right]: x_{i}(s) \in \mathcal{C}\left(\left[t_{0}-\right.\right.\right.$ $\left.\left.\left.\tau_{m}, \tau_{0}\right], \mathbf{R}^{n}\right), x_{i}(s)=x_{j}(s), i, j=1,2, \ldots, N\right\}$ as the synchronization manifold for system (2.7).

Definition 2.2 (see [16]). Dynamical network (2.7) is said to be asymptotically synchronized, if for any initial conditions $\phi_{i}(s), \phi_{j}(s) \in C\left(\left[t_{0}-\tau_{m}, t_{0}\right], \mathbf{R}^{n}\right), i, j=1, \ldots, N$, there exist $M>0$, $\varepsilon>0$ and sufficient large $T>0$ such that $\left\|x_{i}(t)-x_{j}(t)\right\| \leq M e^{-\varepsilon t}$ for all $t \geq T$, where $\varepsilon$ and $M$ are said to be the decay rate and the decay coefficient, respectively. Here $\|\cdot\|$ denotes the Euclidean norm.

Due to the communication delay, the array of coupled nodes cannot be decoupled, and the synchronized state is always not the trajectory of an isolated node but a modified one as (2.7). Furthermore, delayed coupling matrix and the degree of the node play the important roles in the synchronized state, which has been illustrated in [21]. In the paper, we give an improved discussion for such synchronization. In the case, system (2.7) reaches 
the synchronization, that is, $x_{1}(t)=\cdots=x_{N}(t)=s(t)$, we can deduce the synchronized state equation

$$
\dot{s}(t)=-\alpha(s(t))[\beta(s(t))-A f(s(t))-B f(s(t-\tau(t)))-\mathbf{I}(t)]-l_{i i} K[s(t-\tau(t))-s(t)],
$$

where $i=1,2, \ldots, N$. Obviously, the synchronization is invariant for the coupled system (2.7). Therefore, to realize complete synchronization, the assumption $l_{11}=\cdots=l_{N N}=l$ has to be imposed on the system (2.7).

\section{Delay-Dependent Synchronization Criteria}

Firstly, together with the Kronecker product in [16-21], we can reformulate the system (2.7) as

$$
\begin{aligned}
\dot{\mathrm{x}}(t)= & -\mathbf{a}(x(t))\left[\mathbf{b}(x(t))-\left(I_{N} \otimes A\right) \mathbf{f}(x(t))-\left(I_{N} \otimes B\right) \mathbf{f}(x(t-\tau(t)))-\mathbf{I}(t)\right]-1\left(I_{N} \otimes K\right) \\
& \times[x(t-\tau(t))-x(t)]+(L \otimes F) x(t)+(L \otimes(K+J)) x(t-\tau(t))
\end{aligned}
$$

with $x(t)=\left[x_{1}^{T}(t), \ldots, \mathrm{x}_{N}^{T}(t)\right]^{T}, \mathbf{a}(\mathrm{x}(t))=\operatorname{diag}\left\{\alpha\left(x_{1}(t)\right), \ldots, \alpha\left(x_{N}(t)\right)\right\}, \mathbf{b}(\mathrm{x}(t))=$ $\left[\beta^{T}\left(x_{1}(t)\right), \ldots, \beta^{T}\left(x_{N}(t)\right)\right]^{T}, \mathbf{f}(\mathbf{x}(\cdot))=\left[f^{T}\left(x_{1}(\cdot)\right), \ldots, f^{T}\left(x_{N}(\cdot)\right)\right]^{T}$, and $\mathbf{I}(t)=\left[I^{T}(t), \ldots, I^{T}(t)\right]^{T}$.

In order to derive our results, the following lemmas are essential for obtaining the synchronization criteria.

Lemma 3.1. Let $D, S$, and $P>0$ be real matrices of appropriate dimensions and $\varepsilon>0$. Then for any vectors $x$ and $y$ with appropriate dimensions, one gets $2 x^{T} D^{T} S y \leq \varepsilon^{-1} x^{T} D^{T} P^{-1} D x+\varepsilon y^{T} S^{T} P S y$.

Lemma 3.2. Let $U=\left[u_{i j}\right]_{N \times N}, P \in \mathbf{R}^{n \times n}, x=\left[x_{1}^{T}, x_{2}^{T}, \ldots, x_{N}^{T}\right]^{T}$, and $y=\left[y_{1}^{T}, y_{2}^{T}, \ldots, y_{N}^{T}\right]^{T}$ with $x_{i}, y_{i} \in \mathbf{R}^{n}, i=1, \ldots, N$. If $U=U^{T}$ and each row sum of $U$ is 0 , then $x^{T}(U \otimes P) y=$ $-\sum_{1 \leq i<j \leq N} u_{i j}\left(x_{i}-x_{j}\right)^{T} P\left(y_{i}-y_{j}\right)$.

Then by utilizing the most improved techniques for achieving the criteria in [30], we state and investigate the global exponential synchronization for the system (3.1).

Theorem 3.3. Supposing that assumptions $\left(A_{1}\right)-\left(A_{4}\right)$ hold, then the dynamical system (3.1) is globally exponentially synchronized, if there exist $n \times n$ matrices $P>0, S>0, Z>0, L_{i}(i=1,2,3,4)$, $n \times n$ matrices $P_{l}>0, Q_{l}>0, R_{l}(l=1,2,3)$ making $\left[\begin{array}{cc}P_{l} & R_{l} \\ * & Q_{l}\end{array}\right] \geq 0, n \times n$ diagonal matrices $R>0$, $Q>0, G>0, E>0, U>0, V>0, W>0, H>0, T_{i}>0(i=1,2), 13 n \times n$ matrices $N_{i}(i=1,2,3)$, and one scalar $\delta>0$ such that, for $1 \leq i<j \leq N$, the LMIs in (3.2) hold

$$
\left[\begin{array}{cccc}
\Omega_{i j}+\$+\$^{T} & \bar{H} & \sqrt{\tau_{0}} N_{1} & \sqrt{\bar{\tau}_{m}} N_{2} \\
* & -\delta I & 0 & 0 \\
* & * & -S & 0 \\
* & * & * & -Z
\end{array}\right]<0, \quad\left[\begin{array}{cccc}
\Omega_{i j}+\$+\$ & \bar{H} & \sqrt{\tau_{0}} N_{1} & \sqrt{\bar{\tau}_{m}} N_{3} \\
* & -\delta I & 0 & 0 \\
* & * & -S & 0 \\
* & * & * & -Z
\end{array}\right]<0,
$$


where $\$=\left[\begin{array}{ll}N_{1}-N_{1}+N_{2}-N_{3} 0_{6 n \cdot 13 n}-N_{2}+N_{3} 0_{3 n \cdot 13 n}\end{array}\right], \bar{H}=\left[\begin{array}{ll}0_{n \cdot 13 n} Q\left(\Lambda^{-1}-\Psi^{-1}\right) & 0_{n \cdot n}\end{array}\right]^{T}$,

$$
\Omega_{i j}=\left[\begin{array}{ccccccccccccc}
\Xi_{11} & 0 & 0 & \Xi_{14} & 0 & 0 & \Xi_{17} & L_{1}^{T} & 0 & \Xi_{1,10} & l L_{1}^{T} K & \Xi_{1,12} & \Xi_{1,13} \\
* & \Xi_{22} & 0 & 0 & \Xi_{25} & 0 & 0 & 0 & 0 & 0 & 0 & 0 & 0 \\
* & * & \Xi_{33} & 0 & 0 & \Xi_{36} & 0 & 0 & 0 & 0 & 0 & 0 & 0 \\
* & * & * & \Xi_{44} & 0 & 0 & 0 & A^{T} L_{3} & A^{T} L_{4} & 0 & 0 & A^{T} Q^{T} & 0 \\
* & * & * & * & \Xi_{55} & 0 & 0 & 0 & 0 & 0 & 0 & 0 & 0 \\
* & * & * & * & * & \Xi_{66} & 0 & 0 & 0 & 0 & 0 & 0 & 0 \\
* & * & * & * & * & * & \Xi_{77} & -L_{2}^{T} & 0 & \Xi_{7,10} & l L_{2}^{T} K & -\Psi^{-1} R & 0 \\
* & * & * & * & * & * & * & \Xi_{88} & -L_{3}^{T} & 0 & L_{3}^{T} B & -L_{3}^{T} & 0 \\
* & * & * & * & * & * & * & * & \Xi_{99} & 0 & L_{4}^{T} B & -L_{4}^{T} & 0 \\
* & * & * & * & * & * & * & * & * & \Xi_{10,10} & \Xi_{10,11} & \Xi_{10,12} & 0 \\
* & * & * & * & * & * & * & * & * & * & \Xi_{11,11} & B^{T} Q^{T} & 0 \\
* & * & * & * & * & * & * & * & * & * & * & \Xi_{12,12} & 0 \\
* & * & * & * & * & * & * & * & * & * & * & * & -T_{2}
\end{array}\right]
$$

with $\Xi_{11}=P_{2}+l\left(L_{1}^{T} K+K^{T} L_{1}\right)-l_{i j} N\left(L_{1}^{T} F+F^{T} L_{1}\right)-2 \Gamma^{T} G-U \Sigma_{1}-T_{1} \Pi_{1}-T_{2} \Upsilon_{1}, \Xi_{14}=R_{2}+U \Sigma_{2}$, $\Xi_{17}=P-L_{1}^{T}-l_{i j} N F^{T} L_{2}+2 \Pi^{T} R \Psi^{-1}-2 \Gamma^{T} Q \Lambda^{-1}, \Xi_{1,10}=-l L_{1}^{T} K-l_{i j} N L_{1}^{T}(K+J), \Xi_{1,12}=$ $l K^{T} Q \Lambda^{-1}-l_{i j} N F^{T} Q \Lambda^{-1}+G^{T}+T_{1} \Pi_{2}, \Xi_{1,13}=T_{2} \Upsilon_{2}+Q \Lambda^{-1}-R \Psi^{-1}, \Xi_{22}=-P_{2}+P_{1}+P_{3}-W \Sigma_{1}$, $\Xi_{25}=-R_{2}+R_{1}+R_{3}+W \Sigma_{2}, \Xi_{33}=-P_{3}-H \Sigma_{1}, \Xi_{36}=H \Sigma_{2}-R_{3}, \Xi_{44}=-U+Q_{2}, \Xi_{55}=$ $-Q_{2}+Q_{1}+Q_{3}-W, \Xi_{66}=-Q_{3}-H, \Xi_{77}=-L_{2}^{T}-L_{2}+\tau_{0} S+\bar{\tau}_{m} Z, \Xi_{7,10}=-l L_{2}^{T} K-l_{i j} N L_{2}^{T}(K+J)$, $\Xi_{88}=-E+\delta I_{n}, \Xi_{99}=-L_{4}^{T}-L_{4}+\Psi^{T} E \Psi, \Xi_{10,10}=-(1-\mu) P_{1}-V \Sigma_{1}, \Xi_{10,11}=-(1-\mu) R_{1}+V \Sigma_{2}$, $\Xi_{10,12}=-l K^{T} Q \Lambda^{-1}-l_{i j} N(K+J)^{T} Q \Lambda^{-1}, \Xi_{11,11}=-(1-\mu) Q_{1}-V, \Xi_{12,12}=-2 Q^{T}-T_{1}$.

Proof. Firstly, we can represent the system (3.1) as the following form:

$$
\begin{aligned}
\dot{x}(t) & =y(t) \\
y(t) & =z(t)-l\left(I_{N} \otimes K\right)[x(t-\tau(t))-x(t)]+(L \otimes F) x(t)+(L \otimes(K+J)) x(t-\tau(t)), \\
z(t) & =\mathbf{a}(x(t)) w(t) \\
w(t) & =-\mathbf{b}(x(t))+\left(I_{N} \otimes A\right) \mathbf{f}(x(t))+\left(I_{N} \otimes B\right) \mathbf{f}(x(t-\tau(t)))+\mathbf{I}(t) .
\end{aligned}
$$

Based on assumptions (A1) and (A3), and

$$
U=\left[u_{i j}\right]_{N \cdot N}=\left[\begin{array}{ccc}
N-1 & \cdots & -1 \\
\vdots & \ddots & \vdots \\
-1 & \cdots & N-1
\end{array}\right]
$$


we construct the following Lyapunov-Krasovskii functional:

$$
V(x(t))=V_{1}(x(t))+V_{2}(x(t))+V_{3}(x(t))+V_{4}(x(t))+V_{5}(x(t))
$$

where

$$
\begin{aligned}
V_{1}(x(t))= & x^{T}(t)(U \otimes P) x(t)+2[\Theta x(t)-\mathbf{b}(x(t))]^{T}\left(U \otimes R \Psi^{-1}\right) x(t) \\
& +2[\mathbf{b}(x(t))-\Upsilon x(t)]^{T}\left(U \otimes Q \Lambda^{-1}\right) x(t), \\
V_{2}(x(t))= & \int_{t-\tau(t)}^{t-\tau_{0}}\left[\begin{array}{c}
x(s) \\
\mathbf{f}(x(s))
\end{array}\right]^{T}\left(U \otimes\left[\begin{array}{cc}
P_{1} & R_{1} \\
* & Q_{1}
\end{array}\right]\right)\left[\begin{array}{c}
x(s) \\
\mathbf{f}(x(s))
\end{array}\right] \mathrm{ds}, \\
V_{3}(x(t))= & \int_{t-\tau_{0}}^{t}\left[\begin{array}{c}
x(s) \\
\mathbf{f}(x(s))
\end{array}\right]^{T}\left(U \otimes\left[\begin{array}{cc}
P_{2} & R_{2} \\
* & Q_{2}
\end{array}\right]\right)\left[\begin{array}{c}
x(s) \\
\mathbf{f}(x(s))
\end{array}\right] \mathrm{ds}, \\
V_{4}(x(t))= & \int_{t-\tau_{m}}^{t-\tau_{0}}\left[\begin{array}{c}
x(s) \\
\mathbf{f}(x(s))
\end{array}\right]^{T}\left(U \otimes\left[\begin{array}{cc}
P_{3} & R_{3} \\
* & Q_{3}
\end{array}\right]\right)\left[\begin{array}{c}
x(s) \\
\mathbf{f}(x(s))
\end{array}\right] \mathrm{ds}, \\
V_{5}(x(t))= & \int_{-\tau_{0}}^{0} \int_{t+\theta}^{t} \dot{x}^{T}(s)(U \otimes S) \dot{x}(s) d s d \theta+\int_{-\tau_{m}}^{-\tau_{0}} \int_{t+\theta}^{t} \dot{x}^{T}(s)(U \otimes Z) \dot{x}(s) d s d \theta
\end{aligned}
$$

with two diagonal matrices $R>0, Q>0$, and setting $\Theta=\operatorname{diag}\{\underbrace{\Pi, \Pi, \ldots, \Pi}_{N}\}, \Upsilon=$ $\operatorname{diag}\{\underbrace{\Gamma, \Gamma, \ldots, \Gamma}_{N}\}$. Based on (A3) and Lemma 3.1, one can easily verify the definite positiveness of $V_{1}(x(t))$. deduce

Now, by directly calculating $\dot{V}_{1}(x(t))$ along the trajectory of the system (3.1), we can

$$
\begin{aligned}
\dot{V}_{1}(x(t))= & 2 x^{T}(t)(U \otimes P) y(t)+2[\Theta x(t)-\mathbf{b}(x(t))]^{T}\left(U \otimes R \Psi^{-1}\right) y(t)+2[\Theta y(t)-\dot{b}(x(t))]^{T} \\
& \times\left(U \otimes R \Psi^{-1}\right) x(t)+2[\mathbf{b}(x(t))-\Upsilon x(t)]^{T} \\
& \times\left(U \otimes Q \Lambda^{-1}\right) y(t)+2[\dot{b}(x(t))-\Upsilon y(t)]^{T}\left(U \otimes Q \Lambda^{-1}\right) x(t) \\
= & 2 x^{T}(t)(U \otimes P) y(t)+2[\Theta x(t)-\mathbf{b}(x(t))]^{T}\left(U \otimes R \Psi^{-1}\right) y(t)+2 \mathbf{b}^{T}(x(t)) \\
& \times\left(U \otimes Q \Lambda^{-1}\right) y(t)+2 \dot{b}^{T}(x(t))\left(U \otimes\left(Q \Lambda^{-1}-R \Psi^{-1}\right)\right) x(t)+2 y^{T}(t) \\
& \times\left(U \otimes\left(\Pi^{T} R \Psi^{-1}-2 \Gamma^{T} Q \Lambda^{-1}\right)\right) x(t) .
\end{aligned}
$$


Meanwhile by (3.5), it is easy to derive that

$$
\begin{aligned}
2 \mathbf{b}^{T}(x(t))\left(U \otimes Q \Lambda^{-1}\right) y(t)= & 2 \mathbf{b}^{T}(x(t))\left(U \otimes Q \Lambda^{-1}\right) z(t)+2 \mathbf{b}^{T}(x(t))\left(U \otimes Q \Lambda^{-1}\right) \\
\times & {\left[-1\left(I_{N} \otimes K\right)[x(t-\tau(t))-x(t)]\right.} \\
& +(L \otimes F) x(t)+(L \otimes(K+J)) x(t-\tau(t))] .
\end{aligned}
$$

Noting that $U^{1 / 2} \geq 0$ does hold, then with Lemma 3.1 and $\delta>0$, one can estimate $2 \mathbf{b}^{\mathrm{T}}(x(t))\left(U \otimes Q \Lambda^{-1}\right) z(t)$ as

$$
\begin{aligned}
& 2 \mathbf{b}^{\mathrm{T}}(x(t))\left(U \otimes Q \Lambda^{-1}\right) z(t) \\
&=2 \mathbf{b}^{\mathrm{T}}(x(t))\left(U^{1 / 2} \otimes Q\left[\Lambda^{-1}-\alpha^{-1}(x(t))\right]\right)^{\mathrm{T}}\left(U^{1 / 2} \otimes I\right) z(t)+2 \mathbf{b}^{\mathrm{T}}(x(t))(U \otimes Q) w(t) \\
& \leq \delta z^{T}(t)\left(U \otimes I_{n}\right) z(t)+\delta^{-1} \mathbf{b}^{\mathrm{T}}(x(t)) \\
& \times\left(U \otimes\left(\Lambda^{-T}-\Psi^{-T}\right) Q^{T} Q\left(\Lambda^{-1}-\Psi^{-1}\right)\right) \mathbf{b}(x(t))+2 \mathbf{b}^{\mathrm{T}}(x(t)) \\
& \times(U \otimes Q)\left[-\mathbf{b}(x(t))+\left(I_{N} \otimes A\right) \mathbf{f}(x(t))+\left(I_{N} \otimes B\right) \mathbf{f}(x(t-\tau(t))+\mathbf{I}(t)]\right.
\end{aligned}
$$

Now combining with terms (3.12) and (3.13) yields

$$
\begin{aligned}
\dot{V}_{1}(x(t)) \leq & 2 x^{T}(t)(U \otimes P) y(t)+2[\Theta x(t)-\mathbf{b}(x(t))]^{T}\left(U \otimes R \Psi^{-1}\right) y(t)+\delta z^{T}(t)\left(U \otimes I_{n}\right) z(t) \\
& +\delta^{-1} \mathbf{b}^{T}(x(t))\left(U \otimes\left(\Lambda^{-T}-\Psi^{-T}\right) Q^{T} Q\left(\Lambda^{-1}-\Psi^{-1}\right)\right) \mathbf{b}(x(t))+2 \mathbf{b}^{\mathrm{T}}(x(t))(U \otimes Q) \\
& \times\left[-\mathbf{b}(x(t))+\left(I_{N} \otimes A\right) \mathbf{f}(x(t))+\left(I_{N} \otimes B\right) \mathbf{f}(x(t-\tau(t)))+\mathbf{I}(t)\right]+2 \mathbf{b}^{\mathrm{T}}(x(t)) \\
& \times\left(U \otimes Q \Lambda^{-1}\right)\left[-l\left(I_{N} \otimes K\right)[x(t-\tau(t))-x(t)]+(L \otimes F) x(t)+(L \otimes(K+J)) x(t-\tau(t))\right] \\
& +2 \dot{\mathbf{b}}^{\mathrm{T}}(x(t))\left(U \otimes\left(Q \Lambda^{-1}-R \Psi^{-1}\right)\right) x(t)+2 y^{T}(t)\left(U \otimes\left(\Pi^{T} R \Psi^{-1}-2 \Gamma^{T} Q \Lambda^{-1}\right)\right) x(t), \\
\dot{V}_{2}(x(t)) \leq & {\left[x^{T}\left(t-\tau_{0}\right)\left(U \otimes P_{1}\right) x\left(t-\tau_{0}\right)+2 x^{T}\left(t-\tau_{0}\right)\left(U \otimes R_{1}\right) \mathbf{f}\left(x\left(t-\tau_{0}\right)\right)+\mathbf{f}^{\mathrm{T}}\left(x\left(t-\tau_{0}\right)\right)\right.} \\
& \left.\times\left(U \otimes Q_{1}\right) \mathbf{f}\left(x\left(t-\tau_{0}\right)\right)\right] \\
& \quad(1-\mu)\left[x^{T}(t-\tau(t))\left(U \otimes P_{1}\right) x(t-\tau(t))+2 x^{\mathrm{T}}(t-\tau(t))\left(U \otimes R_{1}\right) \mathbf{f}(x(t-\tau(t))),\right. \\
& \left.\quad+\mathbf{f}^{\mathrm{T}}(x(t-\tau(t)))\left(U \otimes Q_{1}\right) \mathbf{f}(x(t-\tau(t)))\right]
\end{aligned}
$$




$$
\begin{aligned}
& \dot{V}_{3}(x(t))= {\left[x^{T}(t)\left(U \otimes P_{2}\right) x(t)+2 x^{T}(t)\left(U \otimes R_{2}\right) \mathbf{f}(x(t))+\mathbf{f}^{\mathrm{T}}(x(t))\left(U \otimes Q_{2}\right) \mathbf{f}(x(t))\right] } \\
&- {\left[x^{T}\left(t-\tau_{0}\right)\left(U \otimes P_{2}\right) x\left(t-\tau_{0}\right)+2 x^{T}\left(t-\tau_{0}\right)\left(U \otimes R_{2}\right) \mathbf{f}\left(x\left(t-\tau_{0}\right)\right)\right.} \\
&\left.+\mathbf{f}^{\mathrm{T}}\left(x\left(t-\tau_{0}\right)\right)\left(U \otimes Q_{2}\right) \mathbf{f}\left(x\left(t-\tau_{0}\right)\right)\right], \\
& \dot{V}_{4}(x(t))= {\left[x^{T}\left(t-\tau_{0}\right)\left(U \otimes P_{3}\right) x\left(t-\tau_{0}\right)+2 x^{T}\left(t-\tau_{0}\right)\left(U \otimes R_{3}\right) \mathbf{f}\left(x\left(t-\tau_{0}\right)\right)\right.} \\
&\left.+\mathbf{f}^{\mathrm{T}}\left(x\left(t-\tau_{0}\right)\right)\left(U \otimes Q_{3}\right) \times \mathbf{f}\left(x\left(t-\tau_{0}\right)\right)\right] \\
&-\left[\begin{array}{c}
x^{T}\left(t-\tau_{m}\right)\left(U \otimes P_{3}\right) x\left(t-\tau_{m}\right)+2 x^{T}\left(t-\tau_{m}\right)\left(U \otimes R_{3}\right) \mathbf{f}\left(x\left(t-\tau_{m}\right)\right) \\
\end{array}\right. \\
&\left.+\mathbf{f}^{\mathrm{T}}\left(x\left(t-\tau_{m}\right)\right)\left(U \otimes Q_{3}\right) \mathbf{f}\left(x\left(t-\tau_{m}\right)\right)\right], \\
& \dot{V}_{5}(x(t))= y^{T}(t)\left[\tau_{0}(U \otimes S)+\bar{\tau}_{m}(U \otimes Z)\right] y(t)-\int_{t-\tau_{0}}^{t} y^{T}(s)(U \otimes S) y(s) d s \\
& y^{T}(s)(U \otimes Z) y(s) d s .
\end{aligned}
$$

For any $n \times n$ matrices $L_{i}(i=1,2,3,4)$, it follows from (3.5) and (3.7) that

$$
\begin{aligned}
0=2 & {\left[x^{T}(t)\left(U \otimes L_{1}^{T}\right)+y^{T}(t)\left(U \otimes L_{2}^{T}\right)\right] } \\
& \times\left[-y(t)+z(t)-l\left(I_{N} \otimes K\right)[x(t-\tau(t))-x(t)]+(L \otimes F) x(t)+(L \otimes(K+J)) x(t-\tau(t))\right] \\
& =2\left[z^{T}(t)\left(U \otimes L_{3}^{T}\right)+w^{T}(t)\left(U \otimes L_{4}^{T}\right)\right] \\
& \quad \times\left[-w(t)-\mathbf{b}(x(t))+\left(I_{N} \otimes A\right) \mathbf{f}(x(t))+\left(I_{N} \otimes B\right) \mathbf{f}(x(t-\tau(t)))+\mathbf{I}(t)\right] .
\end{aligned}
$$

By utilizing (A3) and (3.6), for any $n \times n$ diagonal matrices $G \geq 0, E \geq 0$, the following inequality holds

$$
\begin{aligned}
0 \leq & 2\left[\mathbf{b}^{\mathrm{T}}(x(t))(U \otimes G) x(t)-x^{T}(t)\left(U \otimes \Gamma^{T} G\right) x(t)\right] \\
& +\left[w^{T}(t)\left(U \otimes \Psi^{T} E \Psi\right) w(t)-z^{T}(t)(U \otimes E) z(t)\right]
\end{aligned}
$$


Meanwhile, based on (3.14) and (3.19), it is easy to check that $U L=N L$, and

$$
\begin{gathered}
\left(U \otimes Q \Lambda^{-1}\right)(L \otimes F)=(N L) \otimes\left(Q \Lambda^{-1} F\right), \\
\left(U \otimes Q \Lambda^{-1}\right)(L \otimes(K+J))=(N L) \otimes\left(Q \Lambda^{-1}(K+J)\right), \\
\left(U \otimes L_{i}^{T}\right)(L \otimes F)=(N L) \otimes\left(L_{i}^{T} F\right), \quad\left(U \otimes L_{i}^{T}\right)(L \otimes(K+J))=(N L) \otimes\left(L_{i}^{T}(K+J)\right), \\
i=1,2 .
\end{gathered}
$$

Here we can employ the following notations to simplify the subsequent proof

$$
\begin{gathered}
x_{i j}=x_{i}-x_{j}, \quad y_{i j}=y_{i}-y_{j}, \quad z_{i j}=z_{i}-z_{j}, \quad w_{i j}=w_{i}-w_{j}, \\
\beta\left(x_{i j}\right)=\beta\left(x_{i}\right)-\beta\left(x_{j}\right), \quad f\left(x_{i j}\right)=f\left(x_{i}\right)-f\left(x_{j}\right) .
\end{gathered}
$$

Then together with $(U \otimes Q) \mathbf{I}(t)=0$ in (3.14) and $\left(U \otimes L_{3}^{T}\right) \mathbf{I}(t)=\left(U \otimes L_{4}^{T}\right) \mathbf{I}(t)=0$ in (3.19), it follows from Lemma 3.2 and (3.14)-(3.22) that

$$
\begin{aligned}
\dot{V}(x(t)) & \\
\leq-\sum_{1 \leq i<j \leq N}\{ & \mathbf{u}_{i j}\left[2 x_{\mathrm{ij}}^{T}(t) P y_{i j}(t)+2\left[\Pi x_{i j}(t)-\beta\left(x_{i j}(t)\right)\right]\right]^{T} R \Psi^{-1} y_{i j}(t)+\delta z_{i j}^{T}(t) I_{n} z_{i j}(t) \\
+ & \delta^{-1} \beta^{T}\left(x_{i j}(t)\right)\left(\Lambda^{-T}-\Psi^{-T}\right) Q^{T} Q\left(\Lambda^{-1}-\Psi^{-1}\right) \beta\left(x_{i j}(t)\right)+2 \beta^{T}\left(x_{i j}(t)\right) \\
\times & Q\left[-\beta\left(x_{i j}(t)\right)+A f\left(x_{i j}(t)\right)+B f\left(x_{i j}(t-\tau(t))\right)\right]+2 \beta^{T}\left(x_{i j}(t)\right) Q \Lambda^{-1} \\
\times & {\left[-l K\left[x_{i j}(t-\tau(t))-x_{i j}(t)\right]\right]+2 \dot{\beta}^{T}\left(x_{i j}(t)\right)\left(Q \Lambda^{-1}-R \Psi^{-1}\right) x_{i j}(t)+2 y_{i j}^{T}(t) } \\
\times & \left(\Pi^{T} R \Psi^{-1}-2 \Gamma^{T} Q \Lambda^{-1}\right) x_{i j}(t) \\
+ & {\left[x_{i j}^{T}\left(t-\tau_{0}\right)\left(P_{1}-P_{2}+P_{3}\right) x_{i j}\left(t-\tau_{0}\right)+2 x_{i j}^{T}\left(t-\tau_{0}\right)\left(R_{1}-R_{2}+R_{3}\right)\right.} \\
& \left.\times f\left(x_{i j}\left(t-\tau_{0}\right)\right)+f^{T}\left(x_{i j}\left(t-\tau_{0}\right)\right)\left(Q_{1}-Q_{2}+Q_{3}\right) f\left(x_{i j}\left(t-\tau_{0}\right)\right)\right] \\
\times & {\left[x_{i j}^{T}(t-\tau(t)) P_{1} x_{i j}(t-\tau(t))+2 x_{i j}^{T}(t-\tau(t)) R_{1} f\left(x_{i j}(t-\tau(t))\right)\right.} \\
& \left.+f^{T}\left(x_{i j}(t-\tau(t))\right) Q_{1} f\left(x_{i j}(t-\tau(t))\right)\right]
\end{aligned}
$$


Mathematical Problems in Engineering

$$
\begin{aligned}
& \times\left[x_{i j}^{T}(t) P_{2} x_{i j}(t)+2 x_{i j}^{T}(t) R_{2} f\left(x_{i j}(t)\right)+f^{T}\left(x_{i j}(t)\right) Q_{2} f\left(x_{i j}(t)\right)\right] \\
& -\left[x_{i j}^{T}\left(t-\tau_{m}\right) P_{3} x_{i j}\left(t-\tau_{m}\right)+2 x_{i j}^{T}\left(t-\tau_{m}\right) R_{3} f\left(x_{i j}\left(t-\tau_{m}\right)\right)\right. \\
& \left.\quad+f^{T}\left(x_{i j}\left(t-\tau_{m}\right)\right) Q_{3} f\left(x_{i j}\left(t-\tau_{m}\right)\right)\right]+y_{i j}^{T}(t)\left(\tau_{0} S+\bar{\tau}_{m} Z\right) y_{i j}(t) \\
& \quad \int_{t-\tau_{0}}^{t} y_{i j}^{T}(s) S y_{i j}(s) d s-\int_{t-\tau_{m}}^{t-\tau_{0}} y_{i j}^{T}(s) Z y_{i j}(s) d s+2\left[x_{i j}^{T}(t) L_{1}^{T}+y_{i j}^{T}(t) L_{2}^{T}\right] \\
& \times\left[-y_{i j}(t)+z_{i j}(t)-l K\left[x_{i j}(t-\tau(t))-x_{i j}(t)\right]\right] \\
& +2\left[z_{i j}^{T}(t) L_{3}^{T}+w_{i j}^{T}(t) L_{4}^{T}\right]\left[-w_{i j}(t)-\beta\left(x_{i j}(t)\right)+A f\left(x_{i j}(t)\right)+B f\left(x_{i j}(t-\tau(t))\right)\right] \\
& +2\left[\beta^{T}\left(x_{i j}(t)\right) G x_{i j}(t)-x_{i j}^{T}(t) \Gamma^{T} G x_{i j}(t)\right]+\left[w_{i j}^{T}(t) \Psi^{T} E \Psi w_{i j}(t)-z_{i j}^{T}(t) E z_{i j}(t)\right] \\
& +2 N l_{i j}\left[\left[\beta^{T}\left(x_{i j}(t)\right) Q \Lambda^{-1}+x_{i j}^{T}(t) L_{1}^{T}+y_{i j}^{T}(t) L_{2}^{T}\right] F x_{i j}(t)\right. \\
& \left.\left.\quad+\left[\beta^{T}\left(x_{i j}(t)\right) Q \Lambda^{-1}+x_{i j}^{T}(t) L_{1}^{T}+y_{i j}^{T}(t) L_{2}^{T}\right](K+J) x_{i j}(t-\tau(t))\right]\right\} .
\end{aligned}
$$

For any $n \times n$ diagonal matrices $U>0, V>0, W>0, H>0, T_{i}>0(i=1,2)$, and $\Sigma_{i}, \Pi_{i}$, $\Upsilon_{i}(i=1,2)$ in (A3)-(A4), it can be deduced that

$$
\begin{gathered}
0 \leq \sum_{1 \leq i<j \leq N}\left\{-\left[x_{i j}^{T}(t) U \Sigma_{1} x_{i j}(t)-2 x_{i j}^{T}(t) U \Sigma_{2} f\left(x_{i j}(t)\right)+f^{T}\left(x_{i j}(t)\right) U f\left(x_{i j}(t)\right)\right]\right. \\
-\left[x_{i j}^{T}(t-\tau(t)) V \Sigma_{1} \times x_{i j}(t-\tau(t))-2 x_{i j}^{T}(t-\tau(t)) V \Sigma_{2} f\left(x_{i j}(t-\tau(t))\right)\right. \\
\left.\quad+f^{T}\left(x_{i j}(t-\tau(t))\right) V f\left(x_{i j}(t-\tau(t))\right)\right] \\
-\left[x_{i j}^{T}\left(t-\tau_{0}\right) W \Sigma_{1} x_{i j}\left(t-\tau_{0}\right)-2 x_{i j}^{T}\left(t-\tau_{0}\right) W \Sigma_{2} f\left(x_{i j}\left(t-\tau_{0}\right)\right)\right. \\
\left.\quad+f^{T}\left(x_{i j}\left(t-\tau_{0}\right)\right) W f\left(x_{i j}\left(t-\tau_{0}\right)\right)\right] \\
-\left[x_{i j}^{T}\left(t-\tau_{m}\right) H \Sigma_{1} x_{i j}\left(t-\tau_{m}\right)-2 x_{i j}^{T}\left(t-\tau_{m}\right) H \Sigma_{2} f\left(x_{i j}\left(t-\tau_{m}\right)\right)\right. \\
\left.+f^{T}\left(x_{i j}\left(t-\tau_{m}\right)\right) H f\left(x_{i j}\left(t-\tau_{m}\right)\right)\right]
\end{gathered}
$$




$$
\begin{aligned}
& -\left[x_{i j}^{T}(t) T_{1} \Pi_{1} x_{i j}(t)-2 x_{i j}^{T}(t) T_{1} \Pi_{2} \beta\left(x_{i j}(t)\right)+\beta^{T}\left(x_{i j}(t)\right) T_{1} \beta\left(x_{i j}(t)\right)\right] \\
& \left.-\left[x_{i j}^{T}(t) T_{2} \Upsilon_{1} x_{i j}(t)-2 x_{i j}^{T}(t) T_{2} \Upsilon_{2} \dot{\beta}\left(x_{i j}(t)\right)+\dot{\beta}^{T}\left(x_{i j}(t)\right) T_{2} \dot{\beta}\left(x_{i j}(t)\right)\right]\right\} .
\end{aligned}
$$

For any $13 n \times n$ matrices $N_{i}(i=1,2,3)$, it follows from Newton-Leibniz formula that

$$
\begin{aligned}
0=2 \sum_{1 \leq i<j \leq N} \zeta_{i j}^{T}(t) & \left\{N_{1}\left[x_{i j}(t)-x_{i j}\left(t-\tau_{0}\right)-\int_{t-\tau(t)}^{t-\tau_{0}} y_{i j}(s)\right]\right. \\
& +N_{2}\left[x_{i j}\left(t-\tau_{0}\right)-x_{i j}(t-\tau(t))-\int_{t-\tau(t)}^{t-\tau_{0}} y_{i j}(s)\right] \\
& \left.+N_{3}\left[x_{i j}(t-\tau(t))-x_{i j}\left(t-\tau_{m}\right)-\int_{t-\tau_{m}}^{t-\tau(t)} y_{i j}(s)\right]\right\},
\end{aligned}
$$

where

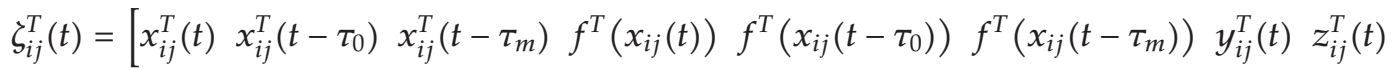

$$
\begin{aligned}
& \left.\times w_{i j}^{T}(t) x_{i j}^{T}(t-\tau(t)) f^{T}\left(x_{i j}(t-\tau(t))\right) \quad \beta^{T}\left(x_{i j}(t)\right) \dot{\beta}^{T}\left(x_{i j}(t)\right)\right] .
\end{aligned}
$$

Now together with the terms (3.24)-(3.26), and $u_{i j}=-1$, we can deduce that

$$
\begin{gathered}
\dot{V}(x(t)) \leq \sum_{1 \leq i<j \leq N} \zeta_{i j}^{T}(t)\left\{\Omega_{i j}+\$+\$^{T}+\delta^{-1} \bar{H} \bar{H}^{T}+\tau_{0} N_{1} S^{-1} N_{1}^{T}\right. \\
\left.+\left[\tau(t)-\tau_{0}\right] N_{2} Z^{-1} N_{2}^{T}+\left[\tau_{m}-\tau(t)\right] N_{3} Z^{-1} N_{3}^{T}\right\} \zeta_{i j}(t) \\
:=\sum_{1 \leq i<j \leq N} \zeta_{i j}^{T}(t) \Delta_{i j}(t) \zeta_{i j}(t),
\end{gathered}
$$

where $\Omega_{i j}, \$$, and $\bar{H}$ are presented in (3.2). Through using Schur-complement and convex combination, the LMIs in (3.2) can guarantee $\Delta_{i j}(t)<0$ and thus, there must exist one scalar $x>0$ such that $\Delta_{i j}(t) \leq-x I<0$. Therefore, one can get

$$
\dot{V}(x(t)) \leq \sum_{1 \leq i<j \leq N} \zeta_{i j}^{T}(t) \Delta_{i j}(t) \zeta_{i j}(t) \leq-\chi\left[\sum_{1 \leq i<j \leq N}\left\|x_{i j}(t)\right\|^{2}+\sum_{1 \leq i<j \leq N}\left\|x_{i j}(t-\tau(t))\right\|^{2}\right],
$$

which indicates that the system (3.1) can reach the global asymptotical synchronization. 
Based on (A1)-(A4), (3.9), and direct computing, there must exist three scalars $\Theta_{i}>$ $0(i=1,2,3)$ such that

$$
V(x(t)) \leq \sum_{1 \leq i<j \leq N}\left[\Theta_{1}\left\|x_{i j}(t)\right\|^{2}+\Theta_{2} \int_{t-\tau_{m}}^{t}\left\|x_{i j}(s)\right\|^{2} d s+\Theta_{3} \int_{t-\tau_{m}}^{t}\left\|x_{i j}(s-\tau(s))\right\|^{2} d s\right] .
$$

Letting $\bar{V}(x(t))=e^{2 k t} V(x(t))$, one can deduce that $\dot{\bar{V}}(x(t))=2 k e^{2 k t} V(x(t))+e^{2 k t} \dot{V}(x(t))$, and

$$
\begin{aligned}
& \bar{V}(x(t))-V(x(0))= \int_{0}^{t} \dot{\bar{V}}(x(s)) d s \\
& \leq \sum_{1 \leq i<j \leq N} \int_{0}^{t} e^{2 k s}\left\{-x\left[\left\|x_{i j}(s)\right\|^{2}+\left\|x_{i j}(s-\tau(s))\right\|^{2}\right]\right. \\
&+2 k\left[\Theta_{1}\left\|x_{i j}(s)\right\|^{2}+\Theta_{2} \int_{s-\tau_{m}}^{s}\left\|x_{i j}(\theta)\right\|^{2} d \theta\right. \\
&\left.\left.+\Theta_{3} \int_{s-\tau_{m}}^{s}\left\|x_{i j}(\theta-\tau(\theta))\right\|^{2} d \theta\right]\right\} d s .
\end{aligned}
$$

By $1 \leq i<j \leq N$ and changing the integration sequences, we have

$$
\begin{aligned}
\int_{0}^{t} e^{2 k s} \int_{s-\tau_{m}}^{s}\left\|x_{i j}(\theta)\right\|^{2} d \theta d s \leq \tau_{m} e^{2 k \tau_{m}} & {\left[\int_{-\tau_{m}}^{0}\left\|x_{i j}(\theta)\right\|^{2} e^{2 k \theta} d \theta+\int_{0}^{t}\left\|x_{i j}(\theta)\right\|^{2} e^{2 k \theta} d \theta\right], } \\
\int_{0}^{t} e^{2 k s} \int_{s-\tau_{m}}^{s}\left\|x_{i j}(\theta-\tau(\theta))\right\|^{2} d \theta d s \leq & \tau_{m} e^{2 k \tau_{m}} \\
\times & {\left[\int_{-2 \tau_{m}}^{0}\left\|x_{i j}(\theta)\right\|^{2} e^{2 k \theta} d \theta+\int_{0}^{t}\left\|x_{i j}(\theta-\tau(\theta))\right\|^{2} e^{2 k \theta} d \theta\right] . }
\end{aligned}
$$

Substituting the terms (3.32) into the relevant ones in (3.31), it is easy to have

$$
\begin{aligned}
\bar{V}(x(t)) \leq V(x(0))+\sum_{1 \leq i<j \leq N}\{ & {\left[2 k \Theta_{1}+2 k \Theta_{2} \tau_{\mathrm{m}} e^{2 k \tau_{\mathrm{m}}}-x\right] \int_{0}^{t}\left\|x_{i j}(\theta)\right\|^{2} e^{2 k \theta} d \theta } \\
+ & {\left.\left[2 k \Theta_{3} \tau_{m} e^{2 k \tau_{m}}-x\right] \int_{0}^{t}\left\|x_{i j}(\theta-\tau(\theta))\right\|^{2} e^{2 k \theta} d \theta+h(k)\right\} }
\end{aligned}
$$

in which $h(k)=2 k \Theta_{2} \tau_{m} e^{2 k \tau_{m}} \int_{-\tau_{m}}^{0}\left\|x_{i j}(\theta)\right\|^{2} d \theta+2 k \Theta_{3} \tau_{m} e^{2 k \tau_{m}} \int_{-2 \tau_{m}}^{0}\left\|x_{i j}(\theta)\right\|^{2} d \theta$. Choose an appropriate scalar $k_{0}>0$ such that $2 k_{0} \Theta_{1}+2 k_{0} \Theta_{2} \tau_{m} e^{2 k_{0} \tau_{m}}-x \leq 0,2 k_{0} \Theta_{3} \tau_{m} e^{2 k_{0} \tau_{m}}-x \leq 0$, 
one has $\bar{V}(x(t)) \leq h\left(k_{0}\right)+V(x(0))$. By directly computing, there must exist a positive scalar $\varpi>0$ such that

$$
h\left(k_{0}\right)+V(x(0)) \leq \varpi \sum_{1 \leq i<j \leq N} \sup _{-2 \tau_{m} \leq s \leq 0}\left\|\phi_{i}(s)-\phi_{j}(s)\right\|^{2} .
$$

Meanwhile, $\bar{V}(x(t)) \geq \lambda_{\min }(P) \sum_{1 \leq i<j \leq N} e^{2 k_{0} t}\left\|x_{i j}(t)\right\|^{2}$. Therefore, it can be deduced that

$$
\left\|x_{i j}(t)\right\| \leq \sqrt{\lambda_{\min }^{-1}(P) \varpi} \cdot \sum_{1 \leq i<j \leq N} \sup _{-2 \tau_{m} \leq s \leq 0}\left\|\phi_{i}(s)-\phi_{j}(s)\right\| e^{-k_{0} t}, \quad \forall t \geq 0 .
$$

By Definition 2.2, the system (3.1) is globally exponentially synchronized, and the proof is completed.

Remark 3.4. Theorem 3.3 presents a novel delay-dependent criterion guaranteeing arrays of coupled Cohen-Grossberg neural networks (2.7) to be globally synchronized. In [16-22], the authors considered global synchronization of an array of coupled neural networks of simple forms and in the paper, we derive a more general delayed neural networks and extended the case to the time variable one, which generalizes the earlier ones. Moreover, the conditions are expressed in terms of LMIs, therefore, by using LMI in Matlab Toolbox, it is straightforward and convenient to check the feasibility of the proposed results without tuning any parameters.

If there does not exist one single delayed coupling in system (2.1), that is, $K=0$, which means that the restriction $l_{11}=l_{22}=\cdots=l_{N N}=l$ in $L=\left[l_{i j}\right]_{N \times N}$ is removed. Then together with the proof of Theorem 3.3, we can derive the following theorem.

Theorem 3.5. Supposing that assumptions $\left(A_{1}\right)-\left(A_{4}\right)$ hold, then the dynamical system (3.1) is globally exponentially synchronized, if there exist $n \times n$ matrices $P>0, S>0, Z>0, L_{i}(i=1,2,3,4)$,

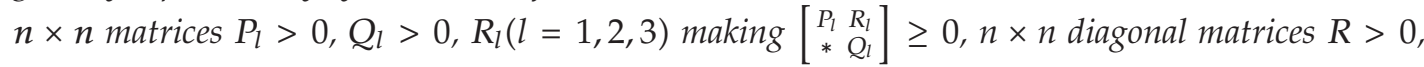
$Q>0, G>0, E>0, U>0, V>0, W>0, H>0, T_{i}>0(i=1,2), 13 n \times n$ matrices $N_{i}(i=1,2,3)$, and one scalar $\delta>0$ such that, for $1 \leq i<j \leq N$, the LMIs in (3.36) hold

$$
\left[\begin{array}{cccc}
\Omega_{i j}+\$+\$ \Phi^{T} & \bar{H} & \sqrt{\tau_{0}} N_{1} & \sqrt{\bar{\tau}_{m}} N_{2} \\
* & -\delta I & 0 & 0 \\
* & * & -S & 0 \\
* & * & * & -Z
\end{array}\right]<0, \quad\left[\begin{array}{cccc}
\Omega_{i j}+\$+\$ \Phi^{T} & \bar{H} & \sqrt{\tau_{0}} N_{1} & \sqrt{\bar{\tau}_{m}} N_{3} \\
* & -\delta I & 0 & 0 \\
* & * & -S & 0 \\
* & * & * & -Z
\end{array}\right]<0,
$$


where $\$=\left[N_{1}-N_{1}+N_{2}-N_{3} 0_{6 n \cdot 13 n}-N_{2}+N_{3} 0_{3 n \cdot 13 n}\right], \bar{H}=\left[\begin{array}{ll}0_{n \cdot 11 n} Q\left(\Lambda^{-1}-\Psi^{-1}\right) 0_{n \cdot n}\end{array}\right]^{T}$,

$$
\Omega_{i j}=\left[\begin{array}{ccccccccccccc}
\Xi_{11} & 0 & 0 & \Xi_{14} & 0 & 0 & \Xi_{17} & L_{1}^{T} & 0 & \Xi_{1,10} & 0 & \Xi_{1,12} & \Xi_{1,13} \\
* & \Xi_{22} & 0 & 0 & \Xi_{25} & 0 & 0 & 0 & 0 & 0 & 0 & 0 & 0 \\
* & * & \Xi_{33} & 0 & 0 & \Xi_{36} & 0 & 0 & 0 & 0 & 0 & 0 & 0 \\
* & * & * & \Xi_{44} & 0 & 0 & 0 & A^{T} L_{3} & A^{T} L_{4} & 0 & 0 & A^{T} Q^{T} & 0 \\
* & * & * & * & \Xi_{55} & 0 & 0 & 0 & 0 & 0 & 0 & 0 & 0 \\
* & * & * & * & * & \Xi_{66} & 0 & 0 & 0 & 0 & 0 & 0 & 0 \\
* & * & * & * & * & * & \Xi_{77} & -L_{2}^{T} & 0 & \Xi_{7,10} & 0 & -\Psi^{-1} R & 0 \\
* & * & * & * & * & * & * & \Xi_{88} & -L_{3}^{T} & 0 & L_{3}^{T} B & -L_{3}^{T} & 0 \\
* & * & * & * & * & * & * & * & \Xi_{99} & 0 & L_{4}^{T} B & -L_{4}^{T} & 0 \\
* & * & * & * & * & * & * & * & * & \Xi_{10,10} & \Xi_{10,11} & \Xi_{10,12} & 0 \\
* & * & * & * & * & * & * & * & * & * & \Xi_{11,11} & B^{T} Q^{T} & 0 \\
* & * & * & * & * & * & * & * & * & * & * & \Xi_{12,12} & 0 \\
* & * & * & * & * & * & * & * & * & * & * & * & -T_{2}
\end{array}\right]
$$

with $\Xi_{11}=P_{2}-l_{i j} N\left(L_{1}^{T} F+F^{T} L_{1}\right)-\Gamma^{T} G-G^{T} \Gamma-U \Sigma_{1}-T_{1} \Pi_{1}-T_{2} \Upsilon_{1}, \Xi_{14}=R_{2}+U \Sigma_{2}, \Xi_{17}=$ $P-L_{1}^{T}-l_{i j} N F^{T} L_{2}+2 \Pi^{T} R \Psi^{-1}-2 \Gamma^{T} Q \Lambda^{-1}, \Xi_{1,10}=-l_{i j} N L_{1}^{T} J, \Xi_{1,12}=-l_{i j} N F^{T} Q \Lambda^{-1}+G^{T}+T_{1} \Pi_{2}$, $\Xi_{1,13}=T_{2} \Upsilon_{2}+Q \Lambda^{-1}-R \Psi^{-1}, \Xi_{22}=-P_{2}+P_{1}+P_{3}-W \Sigma_{1}, \Xi_{25}=-R_{2}+R_{1}+R_{3}+W \Sigma_{2}, \Xi_{33}=$ $-P_{3}-H \Sigma_{1}, \Xi_{36}=H \Sigma_{2}-R_{3}, \Xi_{44}=-U+Q_{2}, \Xi_{55}=-Q_{2}+Q_{1}+Q_{3}-W, \Xi_{66}=-Q_{3}-H$, $\Xi_{77}=-L_{2}^{T}-L_{2}+\tau_{0} S+\bar{\tau}_{m} Z, \Xi_{7,10}=-l_{i j} N L_{2}^{T} J, \Xi_{88}=-E+\delta I_{n}, \Xi_{99}=-L_{4}^{T}-L_{4}+\Psi^{T} E \Psi$, $\Xi_{10,10}=-(1-\mu) P_{1}-V \Sigma_{1}, \Xi_{10,11}=-(1-\mu) R_{1}+V \Sigma_{2}, \Xi_{10,12}=-l_{i j} N J^{T} Q \Lambda^{-1}, \Xi_{11,11}=-(1-\mu) Q_{1}-V$, $\Xi_{12,12}=-2 Q^{T}-T_{1}$.

Proof. Letting $K=0$ in system (2.1) and employing similar methods of proving Theorem 3.3, we can easily derive the theorem and the detailed proof is omitted here.

Remark 3.6. Theorems 3.3-3.5 require the upper bound $\mu$ of time-delay $\tau(t)$ to be known. If $\mu$ is unknown, by setting $P_{1}=R_{1}=Q_{1}$ in (3.9), we can derive the delay-dependent and delay-derivative-independent synchronization criteria for the coupled systems (2.7) based on Theorems 3.3-3.5.

Remark 3.7. Together with the similar proof, we can deal with the global synchronization for arrays of coupled CGNNs with hybrid couplings described in [18]. Moreover, we still can investigate the global synchronization for arrays of coupled uncertain CGNNs with more general forms

$$
\begin{aligned}
\dot{x}_{i}(t)= & -\alpha\left(x_{i}(t)\right)\left[\beta\left(x_{i}(t)\right)-A(t) f\left(x_{i}(t)\right)-B(t) f\left(x_{i}(t-\tau(t))\right)-\mathbf{I}(t)\right] \\
& +\sum_{j=1, j \neq i}^{N} l_{i j}^{1} F\left[x_{j}(t)-x_{i}(t)\right]
\end{aligned}
$$




$$
\begin{aligned}
& +\sum_{j=1, j \neq i}^{N} l_{i j}^{2} K\left[x_{j}(t-\tau(t))-x_{i}(t)\right] \\
& +\sum_{j=1, j \neq i}^{N} l_{i j}^{3} J\left[x_{j}(t-\tau(t))-x_{i}(t-\tau(t))\right],
\end{aligned}
$$

in which $A(t)=A+\Delta A(t), B(t)=B+\Delta B(t)$, and we set $L^{h}=\left[l_{i j}^{h}\right]_{n \times n}$ for $h=, 1,2,3$. Here $\Delta A(t)$ and $\Delta B(t)$ are unknown matrices representing variable parametric uncertainties satisfying

$$
[\Delta A(t) \Delta B(t)]=F_{0} \Delta(t)\left[E_{1} E_{2}\right], \quad \Delta(t)=\Lambda(t)\left(I-J_{0} \Lambda(t)\right)^{-1}, \quad I-J_{0}^{T} J_{0}>0,
$$

in which $F_{0}, J_{0}, E_{i}(i=1,2)$ are known appropriately as dimensional matrices and $\Lambda(t)$ is an unknown variable matrix function satisfying $\Lambda^{T}(t) \Lambda(t) \leq I$. By utilizing the similar methods in Theorems 3.3-3.5, one can easily derive the more general results based on [30, Lemma 5].

Remark 3.8. It is worth pointing out that it is possible to extend our main results to more complex Cohen-Grossberg neural networks, such as CGNNs with distributed delay, stochastic perturbations, and Markovian jumping parameters. The corresponding results will appear in our future works.

\section{Numerical Examples}

In the section, two examples are provided to illustrate the effectiveness of the proposed results.

Example 4.1. We consider the delayed Cohen-Grossberg neural network models described by

$$
\dot{x}(t)=-\alpha(x(t))[\beta(x(t))-A f(x(t))-B f(x(t-\tau(t)))-I(t)]
$$

with $\alpha(x)=\operatorname{diag}\left\{0.8+0.2\left|\cos \left(2 x_{1}\right)\right|, 1.0-0.2\left|\cos \left(2 x_{2}\right)\right|, 0.8+0.2\left|\sin \left(2 x_{3}\right)\right|\right\}$,

$$
\begin{aligned}
& A=\left[\begin{array}{ccc}
-10 & 10 & 2 \\
1 & 0 & 1 \\
0 & -10 & 0
\end{array}\right], \quad B=\left[\begin{array}{ccc}
3 & 2 & -2 \\
2 & -3 & 2 \\
-2 & 2 & 3
\end{array}\right], \quad \beta(x)=\left[\begin{array}{l}
0.9 x_{1}+0.05 \sin \left(2 x_{1}\right) \\
0.9 x_{2}+0.05 \cos \left(2 x_{2}\right) \\
0.9 x_{3}+0.05 \sin \left(2 x_{3}\right)
\end{array}\right], \\
& f(x)=\left[\begin{array}{l}
0.3\left(\left|x_{1}+1\right|-\left|x_{1}-1\right|\right) \\
0.3\left(\left|x_{2}+1\right|-\left|x_{2}-1\right|\right) \\
0.3\left(\left|x_{3}+1\right|-\left|x_{3}-1\right|\right)
\end{array}\right], \quad I(t)=0, \quad \tau(t)=0.4+0.2 \sin (20 t)+0.05 \cos ^{2}(40 t) .
\end{aligned}
$$

One can get $\tau_{0}=0.2, \tau_{m}=0.65, \mu=8$, and the functions $\beta_{i}(\cdot), f_{i}(\cdot)$ satisfy the assumptions (A3) and (A4), respectively. Through setting the inner linking matrix $L=\left[\begin{array}{ccc}-2 & 1 & 1 \\ 1 & -2 & 1 \\ 1 & 1 & -2\end{array}\right]$, we consider 


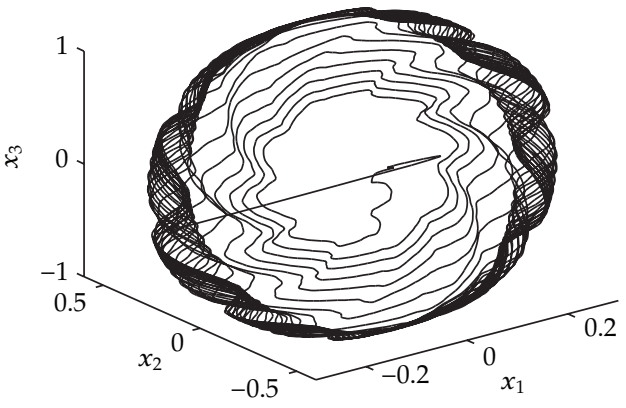

(a)

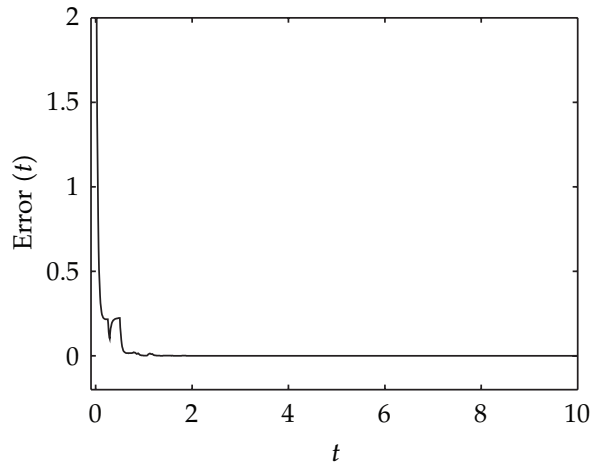

(b)

Figure 1: The synchronized trajectory and total synchronous error of system (4.3).

a dynamical networks comprised of three linearly coupled identical neural network models with time-delay couplings as follows:

$$
\begin{aligned}
\dot{x}_{i}(t)= & -\alpha\left(x_{i}(t)\right)\left[\beta\left(x_{i}(t)\right)-A f\left(x_{i}(t)\right)-B f\left(x_{i}(t-\tau(t))\right)\right]+\sum_{j=1}^{3} l_{i j} F x_{j}(t) \\
& +\sum_{j=1}^{3} l_{i j}(K+J) x_{j}(t-\tau(t))-l K\left[x_{i}(t-\tau(t))-x_{i}(t)\right], \quad i=1,2,3,
\end{aligned}
$$

and choose the coupling matrices as

$$
F=\left[\begin{array}{ccc}
10 & 0 & 0 \\
0 & 10 & 0 \\
0 & 0 & 10
\end{array}\right], \quad K=\left[\begin{array}{ccc}
0.05 & 0 & 0 \\
0 & 0.05 & 0 \\
0 & 0 & 0.05
\end{array}\right], \quad J=\left[\begin{array}{ccc}
0.1 & 0 & 0 \\
0 & 0.1 & 0 \\
0 & 0 & 0.1
\end{array}\right] .
$$

Figure 1 shows that the system has a chaotic attractor. Together with Theorem 3.3 and LMI in Matlab Toolbox, it is easy to check that there exists the feasible solution to the LMIs in (3.2), which can guarantee the array of the system (4.3) to achieve the exponential synchronization. The total error is defined by

$$
\operatorname{error}(t)=\sum_{i=1}^{3} \sqrt{\left[x_{1 i}(t)-x_{2 i}(t)\right]^{2}+\left[x_{2 i}(t)-x_{3 i}(t)\right]^{2}}
$$

and the synchronization error can be seen in Figure 1. During the process of simulation, the initial conditions of nodes are selected as $x_{1}=[-0.5,-0.3,0.3]^{T}, x_{2}=[0.7,-0.5,-0.6]^{T}$, and $x_{3}=[1,0.5,0.3]^{T}$. 


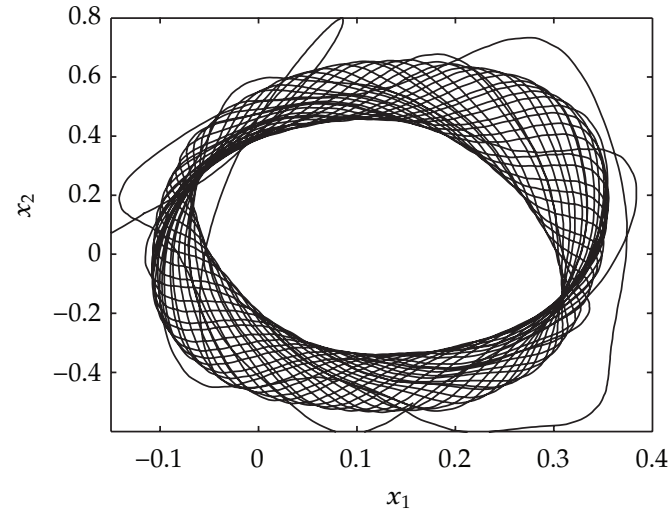

(a)

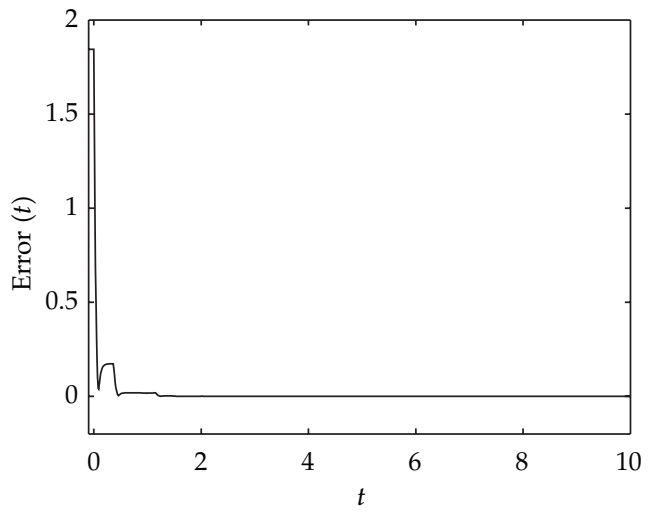

(b)

Figure 2: The synchronized trajectory and total synchronous error of system (4.8).

Example 4.2. Consider one 2-dimensional delayed Cohen-Grossberg neural networks as follows:

$$
\dot{x}(t)=-\alpha(x(t))[\beta(x(t))-A f(x(t))-B f(x(t-\tau(t)))-I(t)],
$$

where $\alpha(x)=\operatorname{diag}\left\{0.9+0.1 \sin \left(x_{1}\right), 0.9+0.1 \cos \left(x_{1}\right)\right\}, \beta(x)=\left[\begin{array}{l}1.1 x_{1}+0.1 \sin \left(x_{1}\right) \\ 1.1 x_{2}+0.1 \sin \left(x_{2}\right)\end{array}\right], A=\left[\begin{array}{cc}2 & -0.2 \\ -0.3 & 3\end{array}\right]$,

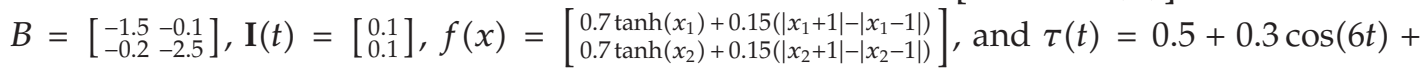
$0.05 \sin ^{2}(40 t)$. Choosing the following inner linking matrix and the coupling matrices, respectively,

$$
L=\left[\begin{array}{ccc}
-3 & 1 & 2 \\
1 & -2 & 1 \\
2 & 1 & -3
\end{array}\right], \quad F=\left[\begin{array}{cc}
10 & 0 \\
0 & 10
\end{array}\right], \quad K=0, \quad J=\left[\begin{array}{cc}
0.1 & 0 \\
0 & 0.1
\end{array}\right]
$$

we still consider a dynamical networks comprised of three coupled identical CGNNs with delayed couplings as

$$
\begin{aligned}
\dot{x}_{i}(t)= & -\alpha\left(x_{i}(t)\right)\left[\beta\left(x_{i}(t)\right)-A f\left(x_{i}(t)\right)-B f\left(x_{i}(t-\tau(t))\right)-I(t)\right] \\
& +\sum_{j=1}^{3} l_{i j} F x_{j}(t)+\sum_{j=1}^{3} l_{i j} K x_{j}(t-\tau(t)) .
\end{aligned}
$$


Then based on Theorem 3.5 and Matlab LMI Toolbox, one can get part feasible solution to (3.36) as follows:

$$
\begin{aligned}
& P=\left[\begin{array}{ll}
2.0635 & 0.0234 \\
0.0234 & 2.2078
\end{array}\right], \quad S=\left[\begin{array}{cc}
0.0274 & -0.0036 \\
-0.0036 & 0.0032
\end{array}\right], \quad Z=\left[\begin{array}{ll}
0.0757 & 0.0016 \\
0.0016 & 0.0870
\end{array}\right], \\
& R=\left[\begin{array}{cc}
0.0415 & 0 \\
0 & 0.0415
\end{array}\right], \quad Q=\left[\begin{array}{cc}
0.0966 & 0 \\
0 & 0.0966
\end{array}\right], \quad G=\left[\begin{array}{cc}
1.2804 & 0 \\
0 & 1.2804
\end{array}\right] \text {, } \\
& E=\left[\begin{array}{cc}
0.0658 & 0 \\
0 & 0.0658
\end{array}\right], \quad U=\left[\begin{array}{cc}
9.4219 & 0 \\
0 & 9.4219
\end{array}\right], \quad V=\left[\begin{array}{cc}
0.6964 & 0 \\
0 & 0.6964
\end{array}\right] \text {, } \\
& W=\left[\begin{array}{cc}
1.1489 & 0 \\
0 & 1.1489
\end{array}\right], \quad H=\left[\begin{array}{cc}
0.6611 & 0 \\
0 & 0.6611
\end{array}\right], \quad T_{1}=\left[\begin{array}{cc}
1.9626 & 0 \\
0 & 1.9626
\end{array}\right] \text {, } \\
& T_{2}=\left[\begin{array}{cc}
1.6523 & 0 \\
0 & 1.6523
\end{array}\right], \quad\left[\begin{array}{ll}
P_{1} & R_{1} \\
R_{1}^{T} & Q_{1}
\end{array}\right]=\left[\begin{array}{cccc}
0.0201 & 0.0013 & -0.0316 & 0.0005 \\
0.0013 & 0.0286 & 0.0003 & -0.0281 \\
-0.0316 & 0.0003 & 0.0621 & -0.0047 \\
0.0005 & -0.0281 & -0.0047 & 0.0289
\end{array}\right] \text {, } \\
& {\left[\begin{array}{ll}
P_{2} & R_{2} \\
R_{2}^{T} & Q_{2}
\end{array}\right]=\left[\begin{array}{cccc}
4.2730 & 0.8822 & -0.4717 & -0.0002 \\
0.8822 & 10.2251 & 0.0277 & -0.3621 \\
-0.4717 & 0.0277 & 2.6380 & 0.0486 \\
-0.0002 & -0.3621 & 0.0486 & 0.7271
\end{array}\right]} \\
& {\left[\begin{array}{ll}
P_{3} & R_{3} \\
R_{3}^{T} & Q_{3}
\end{array}\right]=\left[\begin{array}{cccc}
2.0131 & 0.3881 & -0.2455 & 0.0047 \\
0.3881 & 4.6532 & 0.0180 & -0.2932 \\
-0.2455 & 0.0180 & 1.5619 & 0.0179 \\
0.0047 & -0.2932 & 0.0179 & 0.9050
\end{array}\right]}
\end{aligned}
$$

which means that the global exponential synchronization is achieved for system (4.8). The total error of the array of the system (4.8) is defined by

$$
\operatorname{error}(t)=\sum_{i=1}^{2} \sqrt{\left[x_{1 i}(t)-x_{2 i}(t)\right]^{2}+\left[x_{2 i}(t)-x_{3 i}(t)\right]^{2}}
$$

and the synchronization state and total synchronous error can be depicted in Figure 2 with the initial conditions $x_{1}=[-0.5,-0.3]^{T}, x_{2}=[0.3,0.7]^{T}$, and $x_{3}=[-0.5,-0.6]^{T}$. Moreover, if we choose $\alpha(x)=\operatorname{diag}\left\{0.8+0.2\left(e^{x_{1}} / 1+e^{x_{1}}\right), 0.8+0.2\left(e^{x_{2}} / 1+e^{x_{2}}\right)\right\}$, and $\tau(t)=0.5+0.3 \sin (20 t)+$ $0.05 \cos ^{2}(30 t)$, then the synchronization state and total synchronous error can be described in Figure 3. 


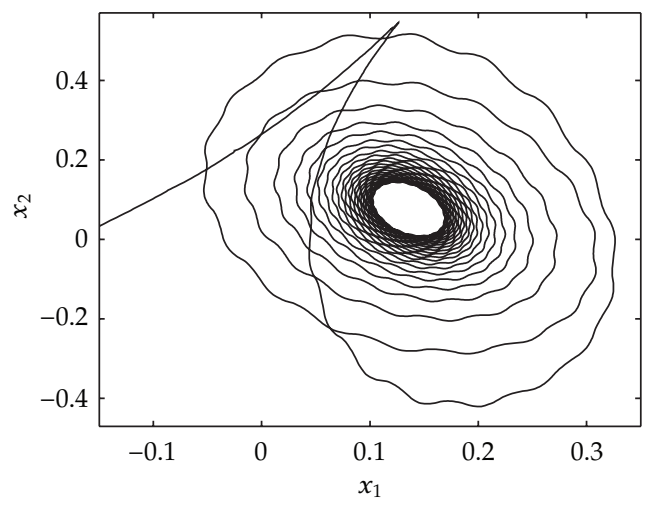

(a)

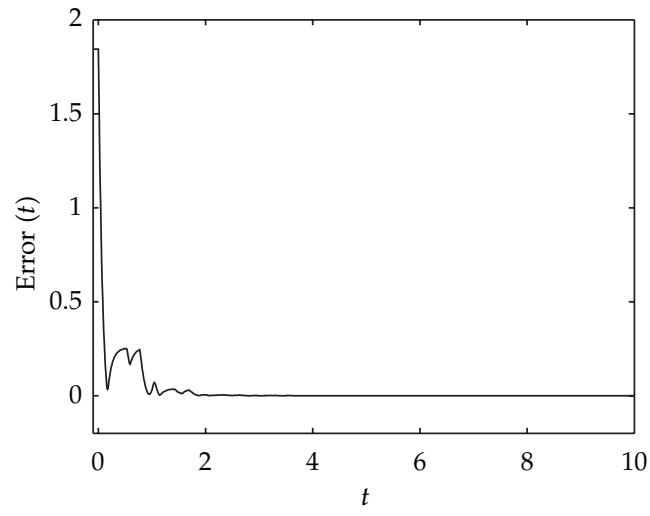

(b)

Figure 3: The synchronized trajectory and total synchronous error of system (4.8).

\section{Conclusions}

This paper has investigated the global exponential synchronization for the coupled CohenGrossberg neural networks with both delayed couplings and time-varying delay. Two novel conditions have been derived by employing Lyapunov-Krasovskii functional and convex combination. It is worth pointing out that, the addressed systems can include many neural network models as its special cases and some good mathematical techniques have been employed, which improve and extend those present results. The derived synchronization criteria are presented in terms of LMIs, which can be checked easily by resorting to Matlab LMI Toolbox. Finally, two numerical examples are utilized to illustrate the effectiveness of the derived methods based on the simulation results.

\section{Acknowledgment}

This work is supported by the national Natural Science Foundation of China nos. 60875035, 60804017, 60904023, 61004032, the Special Foundation of China Postdoctoral Science Funded Project no. 201003546, Jiangsu Planned Projects for Postdoctoral Research Funds no. 0901005B, and China Postdoctoral Science Foundation Funded Project no. 200904501033.

\section{References}

[1] L. M. Pecora and T. L. Carroll, "Synchronization in chaotic systems," Physical Review Letters, vol. 64, no. 8, pp. 821-824, 1990.

[2] T. Carroll and L. Pecora, "Synchronization chaotic circuits," IEEE Transactions on Circuits and Systems. I, vol. 38, no. 4, pp. 453-456, 1991.

[3] C. W. Wu and L. O. Chua, "Application of graph theory to the synchronization in an array of coupled nonlinear oscillators," IEEE Transactions on Circuits and Systems. I, vol. 42, no. 8, pp. 494-497, 1995.

[4] A. Zheleznyak and L. Chua, "Coexistence of low-and high dimensional spatiotemporal chaos in a chain of dissipatively coupled Chua's circuits," International Journal of Bifurcations and Chaos, vol. 4, no. 3, pp. 639-674, 1994.

[5] V. P. Munuzuri and V. P. Villar, "Spiral waves on a 2-D array of nonlinear circuits," IEEE Transactions on Circuits and Systems I, vol. 40, no. 11, pp. 872-877, 1993. 
[6] M. Chen, C.-S. Jiang, Q.-X. Wu, and W.-H. Chen, "Maintaining synchronization by decentralized feedback control in time delay neural networks with parameter uncertainties," International Journal of Neural Systems, vol. 17, no. 2, pp. 115-122, 2007.

[7] Y. Tang, J.-A. Fang, and Q.-Y. Miao, "Synchronization of stochastic delayed neural networks with markovian switching and its application," International Journal of Neural Systems, vol. 19, no. 1, pp. 43-56, 2009.

[8] D. V. Senthilkumar, J. Kurths, and M. Lakshmanan, "Stability of synchronization in coupled timedelay systems using Krasovskii-Lyapunov theory," Physical Review E, vol. 79, no. 6, pp. 1-4, 2009.

[9] R. Follmann, E. E. N. Macau, and E. Rosa, Jr., "Detecting phase synchronization between coupled non-phase-coherent oscillators," Physics Letters. A, vol. 373, no. 25, pp. 2146-2153, 2009.

[10] A. Tarai, S. Poria, and P. Chatterjee, "Synchronization of bidirectionally coupled chaotic Chen's system with delay," Chaos, Solitons and Fractals, vol. 41, no. 1, pp. 190-197, 2009.

[11] W. Wu, W. Zhou, and T. Chen, "Cluster synchronization of linearly coupled complex networks under pinning control," IEEE Transactions on Circuits and Systems. I, vol. 56, no. 4, pp. 829-839, 2009.

[12] W. Wu and T. Chen, "Global synchronization criteria of linearly coupled neural network systems with time-varying coupling," IEEE Transactions on Neural Networks, vol. 19, no. 2, pp. 319-332, 2008.

[13] Y. P. Zhang and J. T. Sun, "Robust synchronization of coupled delayed neural networks under general impulsive control," Chaos, Solitons and Fractals, vol. 41, no. 3, pp. 1476-1480, 2009.

[14] Y. Xia, Z. Yang, and M. Han, "Synchronization schemes for coupled identical Yang-Yang type fuzzy cellular neural networks," Communications in Nonlinear Science and Numerical Simulation, vol. 14, no. 9-10, pp. 3645-3659, 2009.

[15] X. Lou and B. Cui, "Synchronization of neural networks based on parameter identification and via output or state coupling," Journal of Computational and Applied Mathematics, vol. 222, no. 2, pp. 440-457, 2008.

[16] Q. Song, "Synchronization analysis of coupled connected neural networks with mixed time delays," Neurocomputing, vol. 72, no. 16-18, pp. 3907-3914, 2009.

[17] K. Yuan, "Robust synchronization in arrays of coupled networks with delay and mixed coupling," Neurocomputing, vol. 72, no. 4-6, pp. 1026-1031, 2009.

[18] J. D. Cao, G. R. Chen, and P. Li, “Global synchronization in an array of delayed neural networks with hybrid coupling," IEEE Transactions on Systems, Man, and Cybernetics. Part B, vol. 38, no. 2, pp. 488-498, 2008.

[19] W. W. Yu, J. D. Cao, and J. Lü, “Global synchronization of linearly hybrid coupled networks with time-varying delay," SIAM Journal on Applied Dynamical Systems, vol. 7, no. 1, pp. 108-133, 2008.

[20] Z. Fei, H. Gao, and W. X. Zheng, "New synchronization stability of complex networks with an interval time-varying coupling delay," IEEE Transactions on Circuits and Systems II, vol. 56, no. 6, pp. 499-503, 2009.

[21] W. He and J. Cao, "Global synchronization in arrays of coupled networks with one single timevarying delay coupling," Physics Letters. Section A, vol. 373, no. 31, pp. 2682-2694, 2009.

[22] J. D. Cao and L. Li, "Cluster synchronization in an array of hybrid coupled neural networks with delay," Neural Networks, vol. 22, no. 4, pp. 335-342, 2009.

[23] J. L. Liang, Z. D. Wang, and Y. R. Liu, "Robust synchronization of an aray of coupled stochastic discrete-time delayed neural networks," IEEE Transactions on Neural Networks, vol. 19, no. 11, pp. 1910-1921, 2008.

[24] J. L. Liang, Z. D. Wang, Y. R. Liu, and X. Liu, "Global synchronization control of general delayed discrete-time networks with stochastic coupling and disturbances," IEEE Transactions on Systems, Man, and Cybernetics. Part B, vol. 38, no. 4, pp. 1073-1083, 2008.

[25] J. Liang, Z. Wang, and X. Liu, "Global synchronization in an array of discrete-time neural networks with nonlinear coupling and time-varying delays," International Journal of Neural Systems, vol. 19, no. 1, pp. 57-63, 2009.

[26] Z. Chen, "Complete synchronization for impulsive Cohen-Grossberg neural networks with delay under noise perturbation," Chaos, Solitons and Fractals, vol. 42, no. 3, pp. 1664-1669, 2009.

[27] C.-H. Li and S.-Y. Yang, "Synchronization in delayed Cohen-Grossberg neural networks with bounded external inputs," IMA Journal of Applied Mathematics, vol. 74, no. 2, pp. 178-200, 2009.

[28] T. Li, A. Song, and S. Fei, "Synchronization control for arrays of coupled discrete-time delayed CohenGrossberg neural networks," Neurocomputing, vol. 74, no. 1-3, pp. 197-204, 2010. 
[29] M. A. Cohen and S. Grossberg, "Absolute stability of global pattern formation and parallel memory storage by competitive neural networks," Institute of Electrical and Electronics Engineers. Transactions on Systems, Man, and Cybernetics, vol. 13, no. 5, pp. 815-826, 1983.

[30] T. Li, A. G. Song, and S. M. Fei, "Novel stability criteria on discrete-time neural networks with timevarying and distributed delays," International Journal of Neural Systems, vol. 19, no. 4, pp. 269-283, 2009. 


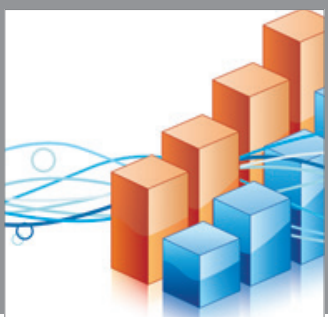

Advances in

Operations Research

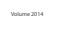

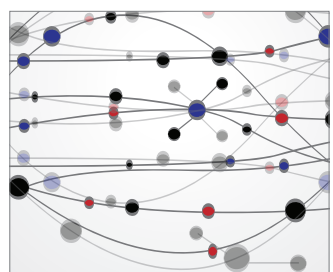

\section{The Scientific} World Journal
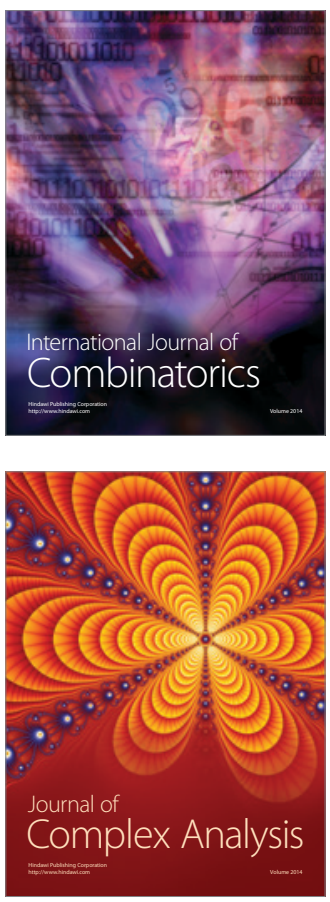

International Journal of

Mathematics and

Mathematical

Sciences
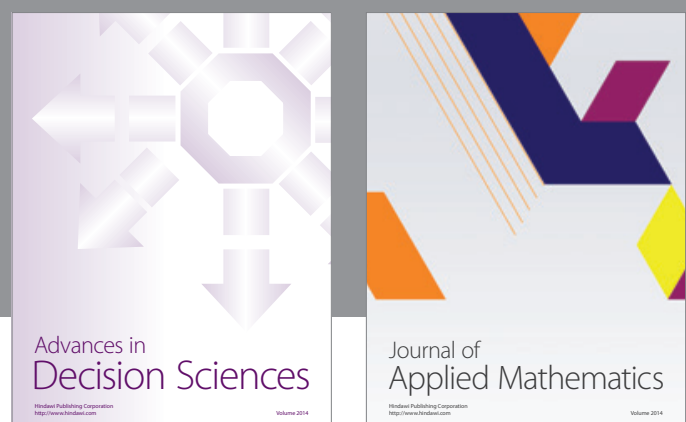

Journal of

Applied Mathematics
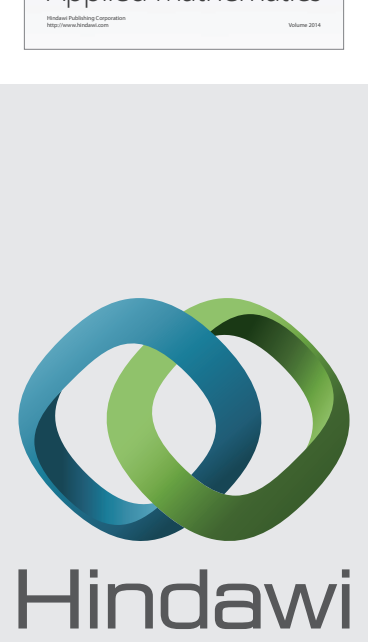

Submit your manuscripts at http://www.hindawi.com
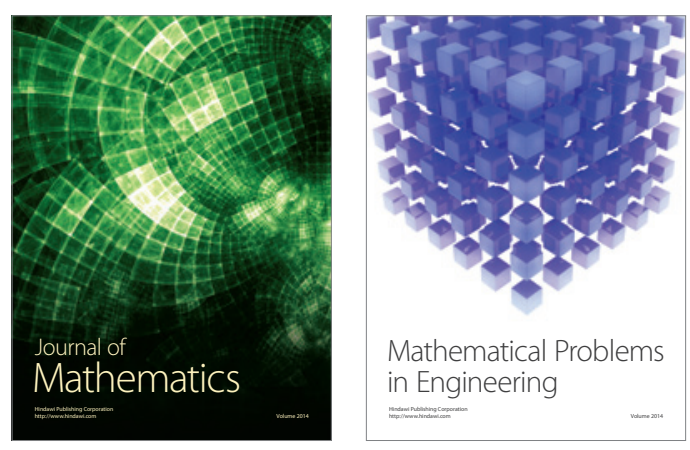

Mathematical Problems in Engineering
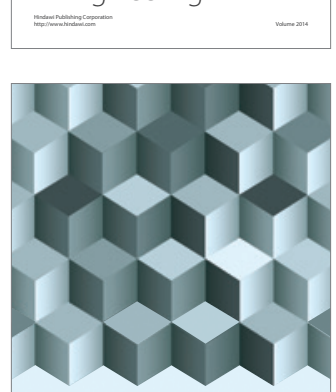

Journal of

Function Spaces
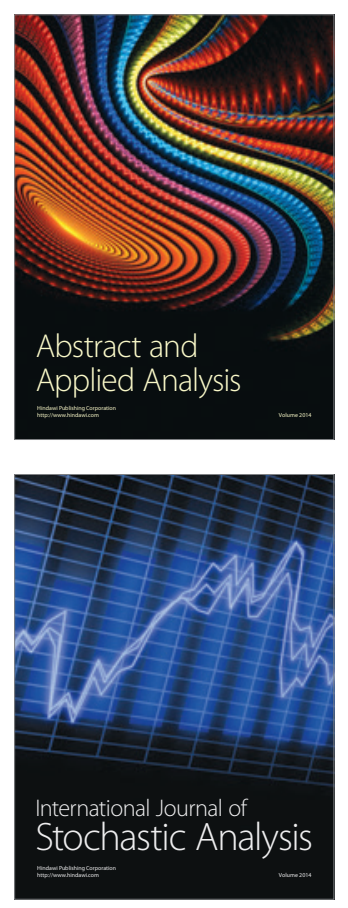

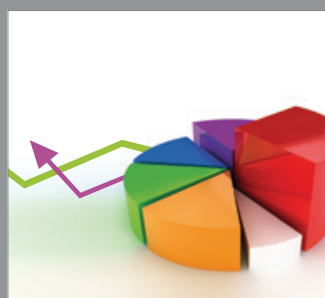

ournal of

Probability and Statistics

Promensencen
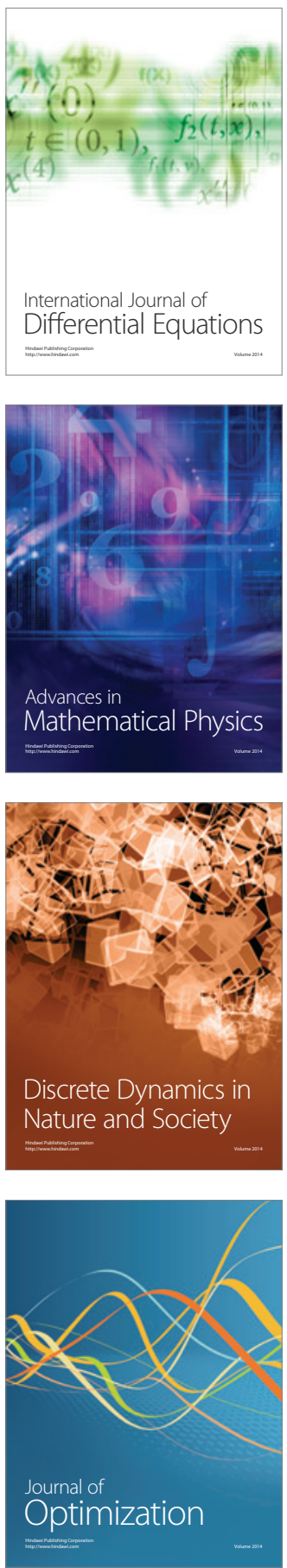